\title{
Anmerkungen zum Artikel «Für den Patienten steht viel auf dem Spiel»
}

\author{
In dem oben genannten Beitrag in der SÄZ 41/2011 haben die Autoren Plattner et \\ al. mögliche Komplikationen bei der Finanzierung ausserkantonaler Spitalbehand- \\ lungen bei Inkrafttreten des neuen Krankenversicherungsgesetzes thematisiert. \\ Die Verfasser des folgenden Beitrags begrüssen die dortigen Ausführungen, regen \\ jedoch einige Änderungen an. Thomas Plattner nimmt dazu Stellung.
}

Beat Kipfera,

Carsten Witzmann ${ }^{b}$

a Facharzt FMH für Herz- und thorakale Gefässchirurgie, Vertrauensarzt KPT

b Rechtsanwalt, EMBA UZH, Leiter Rechtsdienst KPT
Korrespondenz: Dr. med. Beat Kipfer KPT Versicherung Tellstrasse 18 CH-3014 Bern kipfer.beat[at]kpt.ch
Rechtzeitig machen Plattner et al. in ihrem Artikel [1] auf die Problematik der kommenden Finanzierungsänderung im stationären Bereich im Rahmen der Revision des Krankenversicherungsgesetzes (KVG) in verdienstvoller Weise aufmerksam.

Wir möchten es aber nicht unterlassen, einige Bemerkungen anzubringen, damit die ausserordentlich wichtigen Anmerkungen der Autoren korrekt interpretiert werden können. Insbesondere möchten wir Änderungen an der Tabelle anregen:

Die Bestimmungen über die Abgeltung in sogenannten Vertragsspitälern richten sich nach Art. 49a
Abs. 4 KVG (zitiert im Artikel ist Art. 49a Abs. 3 KVG).

Die Beurteilung der Kantonalen Kostengutsprache in Tabelle 1 auf Seite 1586 erfolgt unter dem Gesichtspunkt, ungedeckte Kosten für den Patienten zu vermeiden. Dementsprechend besteht nicht in allen Fällen, die in der Tabelle mit «notwendig» beurteilt werden, auch rechtlich eine Notwendigkeit, eine solche formal einzuholen. Wir würden deshalb empfehlen, die Tabelle wie abgebildet abzuändern. Unsere Änderungsvorschläge sind rot markiert.

Wir sind auch der Meinung, dass eine im rechtlichen Sinne bejahte Notfallbehandlung in einem Ver-

Tabelle 1

Finanzierungsmodalitäten.

\begin{tabular}{|c|c|c|c|c|}
\hline \multirow{2}{*}{$\begin{array}{l}\text { Art. KVG } \\
\text { (neu) }\end{array}$} & \multirow[t]{2}{*}{ Spital } & \multicolumn{2}{|c|}{ Kantonale Kostengutsprache } & \multirow[t]{2}{*}{ Kostenaufteilung } \\
\hline & & Notfall* & Geplanter Eingriff & \\
\hline 41.1 bis & $\begin{array}{l}\text { Spital auf der Liste } \\
\text { des Wohnkantons }\end{array}$ & Nicht notwendig & Nicht notwendig & OKP + Wohnkanton \\
\hline \multirow[t]{2}{*}{$\begin{array}{l}41.1 . \text { bis/ } \\
41.3\end{array}$} & $\begin{array}{l}\text { Spital auf der Liste } \\
\text { des Standortkantons }\end{array}$ & $\begin{array}{l}\text { Nicht notwendig } \\
\text { (Zur Vermeidung } \\
\text { ungedeckter Kosten } \\
\text { empfohlen) }\end{array}$ & Notwendig & $\begin{array}{l}\text { Notfall/Kostengutsprache bewilligt: } \\
\text { OKP + Wohnkanton } \\
\text { (gem. Tarif Standortkanton) }\end{array}$ \\
\hline & & & & $\begin{array}{l}\text { Kein Notfall/Kostengutsprache abgelehnt } \\
\text { Wahleingriff: OKP + Wohnkanton } \\
\text { (maximal gem. Referenztarif Wohnkanton) } \\
+ \text { Zusatzversicherung oder Patient }\end{array}$ \\
\hline \multirow[t]{2}{*}{ 49a Abs. 4} & \multirow[t]{2}{*}{$\begin{array}{l}\text { Spital auf der Liste } \\
\text { eines Drittkantons** }\end{array}$} & \multirow{2}{*}{$\begin{array}{l}\text { Nicht notwendig } \\
\text { (Zur Vermeidung } \\
\text { ungedeckter Kosten } \\
\text { empfohlen) }\end{array}$} & \multirow{2}{*}{$\begin{array}{l}\text { Keine Kostengutspra- } \\
\text { che durch den Kanton }\end{array}$} & $\begin{array}{l}\text { Notfall/Kostengutsprache bewilligt: } \\
\text { OKP + Wohnkanton }\end{array}$ \\
\hline & & & & $\begin{array}{l}\text { Kein Notfall/Kostengutsprache abgelehnt: } \\
\text { OKP + Zusatzversicherung oder Patient }\end{array}$ \\
\hline \multirow[t]{2}{*}{49 a Abs. 4} & \multirow[t]{2}{*}{ Vertragsspital*** } & \multirow{2}{*}{$\begin{array}{l}\text { Nicht notwendig } \\
\text { (Zur Vermeidung } \\
\text { ungedeckter Kosten } \\
\text { empfohlen) }\end{array}$} & \multirow{2}{*}{$\begin{array}{l}\text { Keine Kostengutspra- } \\
\text { che durch den Kanton }\end{array}$} & $\begin{array}{l}\text { Notfall/Kostengutsprache bewilligt: } \\
\text { OKP + Wohnkanton }\end{array}$ \\
\hline & & & & $\begin{array}{l}\text { Kein Notfall/Kostengutsprache abgelehnt: } \\
\text { OKP + Zusatzversicherung oder Patient }\end{array}$ \\
\hline
\end{tabular}

OKP: Obligatorische Krankenpflegeversicherung

* Behandlung muss unverzüglich erfolgen, und die Verlegung/Einweisung in ein Spital auf der Liste des zivilrechtlichen Wohnsitzkantons ist medizinisch nicht vertretbar.

** Kliniken, die sich weder auf der Liste des Wohnsitzkantons noch auf jener des Standortkantons, aber auf einer Liste eines Drittkantons befinden (z. B. Rehabilitationskliniken, deren Trägerschaft sich ausserhalb des Standortkantons befindet).

*** Spital, welches mit einem oder mehreren Versicherern Verträge hat, aber auf keiner kantonalen Liste steht. 


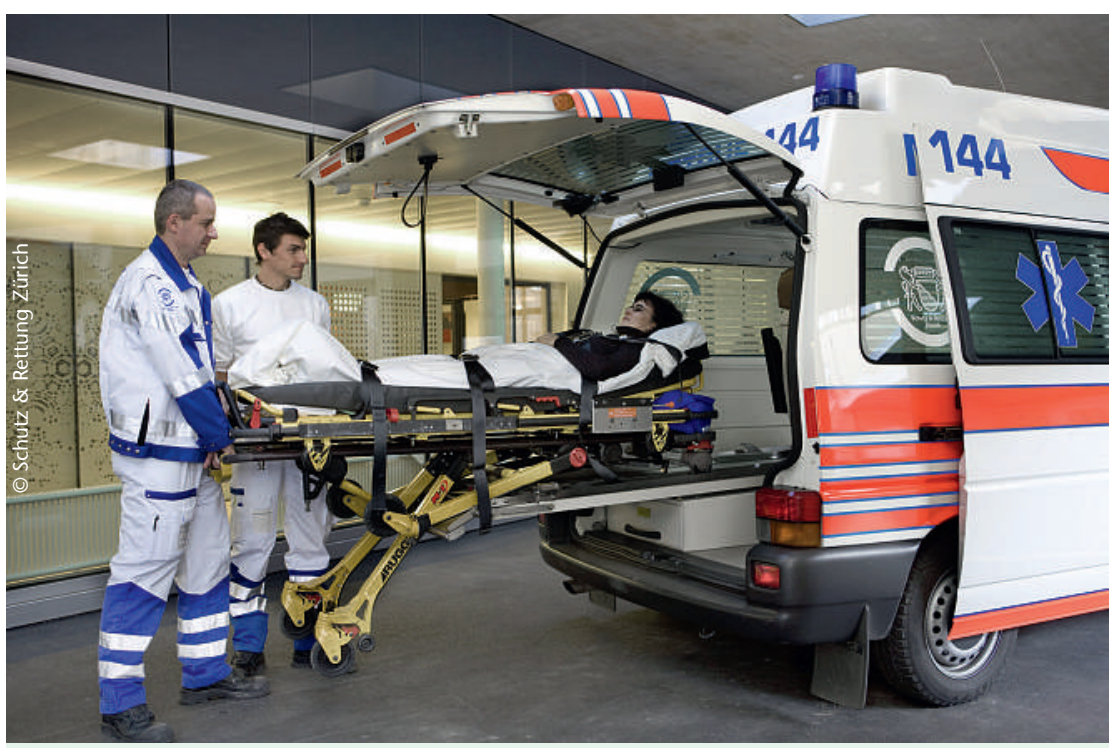

Bei einer ausserkantonalen Hospitalisation sollte im Zweifelsfall ein Kostengutsprachegesuch besser einmal zu viel als zu wenig gestellt werden. gen Kantone beeinflussen, indem sie bei Behandlungen aus medizinischen Gründen in ausserkantonalen Listenspitälern über die Notwendigkeit und damit über die volle Kostenbeteiligungspflicht des Wohnkantons zu entscheiden haben. Die neue Spitalfinanzierung hat aus unserer Sicht weiter zur Folge, dass die kantonalen Spitalplanungen und Spitallisten an Bedeutung verlieren: Eine gewollte Ausdünnung des Angebots an Leistungserbringern auf der Spitalliste des Wohnkantons vermag denselben z.B. nicht finanziell zu entlasten, da der OKP-Versicherte den kantonalen Spitalfinanzierungsanteil «mitnimmt», wenn er sich seine Wahlfreiheit zunutze macht und sich in einem auf der Spitalliste des Standortkantons aufgeführten Listenspital ausserkantonal behandeln lässt (Art. 41 Abs. 1bis Satz 1 KVG). Eine kantonale Spitalplanung, die auf Angebots- und Mengenbeschränkung fokussiert ist, könnte sich dabei als «Rohrkrepierer» erweisen, da der Kantonseinwohner seine medizinischen Bedürfnisse neu auch bei Wahleingriffen ausserkantonal - unter Kostenbeteiligung seines Wohnkantons (!) - befriedigen kann. Ein von ihm allenfalls $\mathrm{zu}$ tragendes finanzielles Risiko (Art. 41 Abs. 1 bis Satz 2 KVG) wird verhältnismässig bescheiden bleiben bzw. lässt sich durch eine entsprechende Zusatzversicherung (Spitalversicherung allgemeine Abteilung ganze Schweiz) abdecken. Bei völlig fehlendem medizinischem Angebot im Wohnkanton hat dieser wie bis anhin eine volle Kostenbeteiligungspflicht (Art. 41 Abs. 3bis lit. b KVG).

\section{Literatur}

1 Plattner T, Wicki Martin M, Gmür R, Lee CY. Für Patienten steht viel auf dem Spiel - auch nach dem 1. Januar 2012. Schweiz Ärztezeitung. 2011;92(41):1585-7.

2 Witzmann C, Kipfer B. Ausserkantonale Hospitalisationen. Kommentar zum Beitrag von Plattner/Lee. Schweiz Ärztezeitung. 2010;91(13/14):526.

\section{Stellungnahme}

Wir danken Dr. med. Kipfer für seine Korrekturen und Ergänzungen. Was die Inhalte der Tabelle betrifft, haben wir in unserem Artikel bereits angedeutet, dass es sich dabei um den Stand des gegenwärtigen (Un-)Wissens handelt. Es kann somit durchaus sein, dass sich gewisse Abläufe im Detail anders darstellen werden. Was die Notwendigkeit einer Kostengutsprache bei Notfällen betrifft, sind sich selbst die Rechtsexperten uneins. Unsere Hinweise richten sich in erster Linie an die Ärzte in Praxis und Spital, und hier ist uns wichtig zu betonen, dass, wie Kollege Kipfer dies auch empfiehlt, im Interesse des Patienten im Zweifelsfall lieber einmal ein Kostengutsprachegesuch zu viel als zu wenig an den Kanton gerichtet wird.

Dr. med. Thomas Plattner, Facharzt für Rechtsmedizin, Stellvertretender Kantonsarzt Fribourg 